\title{
Long non-coding RNA LINC00173 enhances cisplatin resistance in hepatocellular carcinoma via the microRNA-641/RAB14 axis
}

\author{
GUANGLIANG ZHAO ${ }^{1}$, ANHUA ZHANG ${ }^{2}$, SHUFANG SUN $^{3}$ and YUNLONG DING ${ }^{1}$ \\ ${ }^{1}$ Department of Emergency General Surgery, Weifang People's Hospital, Weifang, Shandong 261000; \\ ${ }^{2}$ Department of Gastrointestinal Surgery, Gaomi People's Hospital, Gaomi, Shandong 261500; \\ ${ }^{3}$ Department of Anesthesia, Weifang Maternal and Child Health Hospital, Weifang, Shandong 261000, P.R. China
}

Received August 5, 2020; Accepted January 29, 2021

DOI: $10.3892 / \mathrm{ol} .2021 .12632$

\begin{abstract}
A growing body of evidence indicates that long non-coding RNAs (lncRNAs) play crucial roles in the chemoresistance of human cancers. However, the molecular mechanisms underlying the functions of certain lncRNAs in the chemotherapeutic resistance of hepatocellular carcinoma (HCC) remain unclear. The aim of the present study was to investigate the function and potential mechanism of action of lncRNA LINC00173 in HCC cisplatin (DDP) resistance. Reverse transcription-quantitative PCR analysis indicated that LINC00173 was highly expressed in DDP-resistant HCC tissues and cell lines, and high expression levels of LINC00173 were found to be associated with poor prognosis in patients with HCC. Moreover, LINC00173-knockdown improved the DDP sensitivity of DDP-resistant HCC cells. A luciferase reporter assay also demonstrated that microRNA (miR)-641 was a direct target of LINC00173. miR-641 inhibition restored the promoting effect of LINC00173 knockdown on DDP sensitivity in HCC cells. Furthermore, RAB14 was identified as a target of miR-641, and RAB14 overexpression restrained the inducing effect of LINC00173 knockdown on HCC cell DDP sensitivity. The findings of the present study demonstrated that LINC00173 increased DDP resistance in HCC via the miR-641/RAB14 axis, which may represent a promising therapeutic strategy for HCC.
\end{abstract}

\section{Introduction}

Hepatocellular carcinoma (HCC) is the third most common cause of cancer-associated mortality worldwide (1). Despite advances in diagnosis and treatment, the prognosis of patients with HCC remains unsatisfactory (2). Thus, the pathogenesis

Correspondence to: Professor Yunlong Ding, Department of Emergency General Surgery, Weifang People's Hospital, 151 Guangwen, Kuiwen, Weifang, Shandong 261000, P.R. China E-mail: dingyunlong1980@163.com

Key words: hepatocellular carcinoma, LINC00173, microRNA-641, RAB14, cisplatin of HCC must be further elucidated in order to identify efficient targets for HCC therapy.

Long non-coding RNAs (lncRNAs) are a class of RNA transcripts $>200$ nucleotides (nt) in length that have no protein-coding ability $(3,4)$. Emerging evidence has shown that lncRNAs are involved in the tumorigenesis of multiple different cancer types, including HCC (5). For example, lncRNA DLEU2 promoted HCC cell proliferation and metastasis by interacting with enhancer of zeste 2 polycomb repressive complex 2 subunit (6). 1ncRNA MEG3 suppressed the proliferation of HCC cells by regulating the miR-9-5/SOX11 axis (7), and lncRNA SNHG7 facilitated HCC progression by sponging miR-122-5p and upregulating ribosomal protein L4 (8). Additionally, abnormal expression of IncRNAs is reportedly involved in the resistance of various tumors to chemotherapy, such as breast and gastric cancer $(9,10)$. Numerous studies have indicated that lncRNA LINC00173 functions as an oncogene in the progression of human cancers. For example, LINC00173 was found to regulate cervical cancer cell viability and invasiveness via the miR-182-5p/F-box and the WD repeat domain-containing 7 axes (11). Furthermore, IncRNA LINC00173 accelerated breast cancer progression by downregulating miR-490-3p (12), and accelerated the development of melanoma by upregulating insulin receptor substrate 4 via miRNA-493 sponging (13). However, the function of LINC00173 in HCC chemoresistance remains unknown.

MicroRNAs (miRNAs/miRs) are non-coding RNAs that are $\sim 22 \mathrm{nt}$ in length and play key regulatory roles in the development and chemoresistance of human cancers (14). For example, Niu et al (15) revealed that miRNA-654-3p enhanced cisplatin (DDP) sensitivity by downregulating quinolinate phosphoribosyl transferase and suppressing the PI3K/AKT pathway in ovarian cancer. Yang et al (16) suggested that miRNA-181a-5p promoted the sensitivity of esophageal adenocarcinoma cells to DDP by targeting $\mathrm{Cbl}$ proto-oncogene B. Furthermore, silencing of circRNA CDR1as inhibited the stemness of DDP-resistant non-small cell lung cancer cells by repressing homeobox A9 as a result of miR-641 upregulation (17), suggesting that miR-641 may play an important role in the chemoresistance of cancer cells. However, the mechanisms of miR-641 in the DDP-associated resistance of HCC remain unclear. 
The aim of the present study was to determine whether LINC00173 is upregulated in DDP-resistant HCC tissues and cell lines, and to investigate whether LINC00173 induced the DDP resistance of HCC cells by sponging miR-641 and upregulating RAB14, in order to determine whether the LINC00173/miR-641/RAB14 axis may serve as a novel target for the treatment of HCC.

\section{Materials and methods}

Tissue samples. A total of 30 pairs of HCC and adjacent-normal tissues were acquired from patients at the Weifang People's Hospital (Weifang, China) between February 2018 and November 2019, of which the clinical characteristics are presented in Table I. The inclusion criteria for the patients were: i) Diagnosed with HCC; and ii) not received preoperative radiotherapy, chemotherapy or other adjuvant treatments. The exclusion criteria for the patients were: i) Diagnosed with any other diseases apart from $\mathrm{HCC}$; and ii) failure to cooperate with researchers. The tumor tissues were then divided into DDP-sensitive $(n=11)$ and DDP-resistant $(n=19)$ tissues according to the Response Evaluation Criteria in Solid Tumors (18). All fresh tissues were preserved in liquid nitrogen until use. Written informed consent was obtained from all participants and the study protocol was approved by the Ethics Committee of Weifang People's Hospital.

Cell culture. Human HCC cells (Huh7 and Hep3B) were purchased from the American Type Culture Collection and were incubated with gradually increasing concentrations of DDP for 12 months to obtain DDP-resistant Huh7 (Huh7/DDP) and DDP-resistant Hep3B (Hep3B/DDP) cell lines. The cells were subsequently cultured in RPMI-1640 medium (Invitrogen; Thermo Fisher Scientific, Inc.) supplemented with $10 \%$ FBS (Gibco; Thermo Fisher Scientific, Inc.) at $37^{\circ} \mathrm{C}$ $\left(5 \% \mathrm{CO}_{2}\right)$.

Transfection. The small short hairpin (sh) RNA targeting LINC00173 (10 nM; shLINC00173; 5'-UCCGGGUAGCUA UAUCAUGCG-3') and its negative control (10 nM; shNC; 5'-GAUUAGUUCACAUGCGAUCCU-3'), the miR-641 mimics (10 nM; 5'-GACGAUCCUCUGCAUCGAGUA-3') and scrambled negative control (10 nM; NC mimics; 5'-UAG UUGACGCUUGACGCUAGU-3'), and the miR-641 inhibitor (10 nM; 5'-GAUCGGACAUGGUGCUAGCUU-3') and scrambled negative control (NC inhibitor; 5'-AGUCGACGA UAUGCCAGGUCG-3') were all obtained from Shanghai GenePharma Co., Ltd. For LINC00173 and RAB14 overexpression, LINC00173 or RAB14 cDNA were cloned into the pcDNA3.1 vector (Shanghai GenePharma Co., Ltd.). Transfection was performed using Lipofectamine ${ }^{\circledR} 2000$ (Invitrogen; Thermo Fisher Scientific, Inc.) according to the manufacturer's protocol. The transfected cells were used for subsequent experimentation $48 \mathrm{~h}$ post-transfection.

Reverse transcription-quantitative PCR (RT-qPCR). Total RNA was extracted from HCC and adjacent-normal tissues and Huh7 and Hep3B cells using TRIzol ${ }^{\circledR}$ reagent (Invitrogen; Thermo Fisher Scientific, Inc.) according to the manufacturer's protocol. A total of $1 \mu \mathrm{g}$ RNA was reverse transcribed into
Table I. Clinicopathological features of patients with hepatocellular carcinoma.

\begin{tabular}{lc} 
Variable & Cases, $\mathrm{n}$ \\
\hline Age, years & \\
$<60$ & 19 \\
$\geq 60$ & 11 \\
Sex & \\
Male & 17 \\
Female & 13 \\
Liver cirrhosis & \\
No & 22 \\
Yes & 8 \\
Pathological stage & \\
I-II & 16 \\
III-IV & 14 \\
Tumor size, cm & \\
$<5$ & 17 \\
$\geq 5$ & 13 \\
TNM stage & \\
T1+ T2 & 18 \\
T3+ T4 & 12 \\
\hline
\end{tabular}

TNM, Tumor-Node-Metastasis.

cDNA using the PrimeScript RT reagent kit (Invitrogen; Thermo Fisher Scientific, Inc.) according to the manufacturer's protocol. qPCR was performed using the ABI 7500 real-time PCR System (Thermo Fisher Scientific, Inc.) with the SYBR-Green PCR Master Mix kit (Takara Biotechnology Co., Ltd.). The PCR primers used were as follows: LINC00173 forward, 5'-GCCAGCTCTCGGTACCTGGA-3' and reverse, 5'-GGATCGCAACATTCCTGCCAAG-3'; miR-641 forward, 5'-TTATACTCTCACCATTTGGATC-3' and reverse, 5'-TGA CAAGATTTTACATCAAGAA-3'; RAB14 forward, 5'-CGC TCGAGATGGCAACTGCACCATACAAC-3' and reverse, 5'-CGGAATTCCTAGCAGCCACAGCCTTCTC-3'; GAPDH forward, 5'-ACCACAGTCCATGCCATCAC-3' and reverse, 5'-ACCACCCTGTTGCTGTA-3'; and U6 forward 5'-CTT CGGCAGCACATATACT-3' and U6 reverse, 5'-AAAATA TGGAACGCTTCACG-3'. The qPCR thermocycling conditions were as follows: An initial predenaturation step at $94^{\circ} \mathrm{C}$ for $5 \mathrm{~min}$, followed by 40 cycles of denaturation at $95^{\circ} \mathrm{C}$ for $30 \mathrm{sec}$, annealing at $60^{\circ} \mathrm{C}$ for $30 \mathrm{sec}$ and extension at $72^{\circ} \mathrm{C}$ for $20 \mathrm{sec}$. The relative expression of genes was calculated using the $2^{-\Delta \Delta \mathrm{Cq}}$ method (19). GAPDH and U6 were used as the internal controls for LINC00173 and RAB14, or miR-641, respectively.

Drug sensitivity assay. DDP sensitivity was determined using the Cell Counting Kit-8 (CCK-8) assay (Dojindo Molecular Technologies Inc.) based on the manufacturer's instructions to obtain the half maximal inhibitory concentration $\left(\mathrm{IC}_{50}\right)$ value. Briefly, Huh7/DDP and Hep3B/DDP cells were seeded into 96-well plates at a density of $1 \times 10^{4}$ cells per well, and then 
A

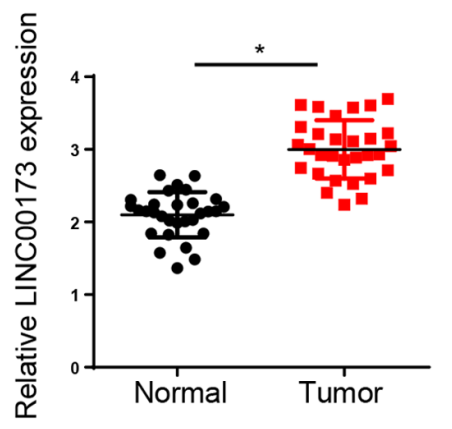

C

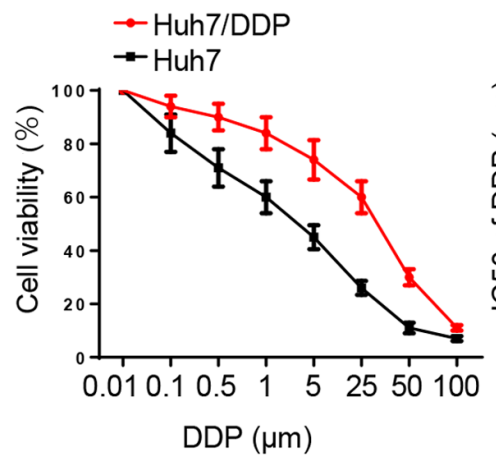

E

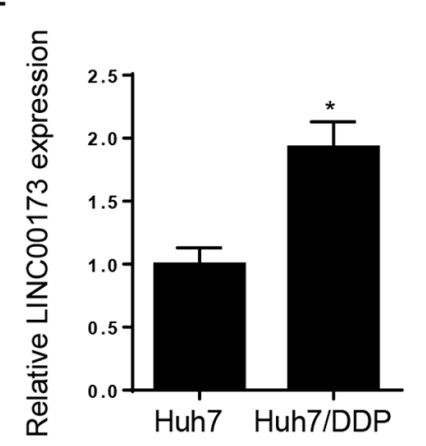

B

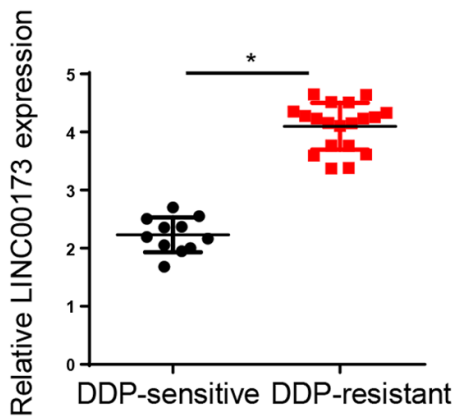

D
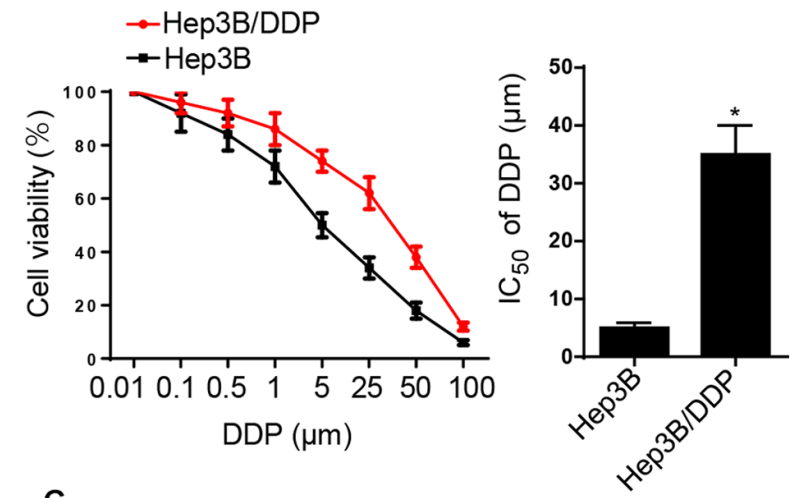

G

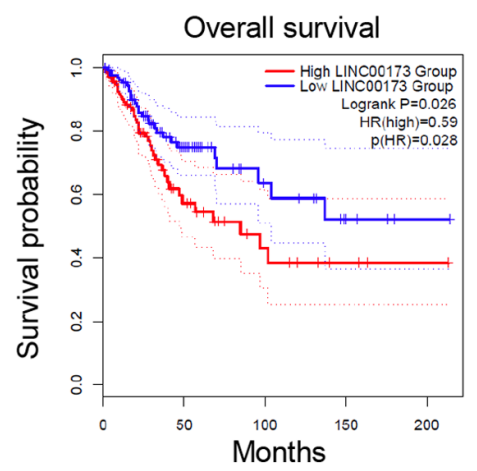

Figure 1. LINC00173 is increased in DDP-resistant HCC tissues and cells. (A) Relative LINC00173 expression levels in HCC tissues and adjacent-normal tissues were determined by RT-qPCR; $n=30$. (B) RT-qPCR analysis of relative LINC00173 expression of in DDP-resistant (Huh7/DDP and Hep3B/DDP) and DDP-sensitive HCC tissues. Cell Counting Kit-8 analysis was used to determine the $\mathrm{IC}_{50}$ value of DDP-resistant (C) Huh7/DDP and (D) Hep3B/DDP cells and their parental cell lines (Huh7 and Hep3B) treated with different concentrations of DDP $(0.01,0.1,0.5,1,5,25,50$ and $100 \mu \mathrm{M})$. RT-qPCR analysis of LINC0017 3expression in DDP-resistant (E) Huh7 and (F) Hep3B cells. (G) Kaplan-Meier survival analysis of the association between LINC00173 expression and the prognosis of patients with HCC. "P<0.05. DDP, cisplatin; HCC, hepatocellular carcinoma; RT-qPCR, reverse transcription-quantitative PCR; IC ${ }_{50}$, half maximal inhibitory concentration; HR, hazard ratio.

treated with DDP for $48 \mathrm{~h}$ at $37^{\circ} \mathrm{C}$. Subsequently, $10 \mu \mathrm{l} \mathrm{CCK}-8$ solution was added to each well and incubated for a further $1 \mathrm{~h}$. The absorbance was recorded using a microplate reader at $450 \mathrm{~nm}$.

Apoptosis analysis. Apoptosis was assessed using the One-Step TUNEL Apoptosis Assay kit (Beyotime Institute of Biotechnology). Briefly, Huh7/DDP and Hep3B/DDP cells $\left(1 \times 10^{4}\right)$ were seeded onto coverslips and then fixed with $4 \%$ paraformaldehyde at $4^{\circ} \mathrm{C}$ for $30 \mathrm{~min}$. The cells were then incubated with TUNEL enzyme for $60 \mathrm{~min}$ at room temperature. Finally, the cells were counterstained with DAPI for $10 \mathrm{~min}$ at room temperature to visualize the cell nuclei. Images were captured from four fields of view using a fluorescence microscope (magnification, $\mathrm{x} 200$ ).
Bioinformatics analysis. The StarBase database (http:// starbase.sysu.edu.cn/) was used to predict interactions between miR-641 and LINC00173 or RAB14 by screening for potential binding sites.

Luciferase reporter assay. The wild-type (wt) or mutant (mut) sequences of LINC00173 and RAB14 were cloned into pmirGLO vectors (Promega Corporation). Then, DDP-resistant HCC cells were co-transfected with miR-641 mimics or $\mathrm{NC}$ mimics and the aforementioned reporter vectors using Lipofectamine 2000 (Invitrogen; Thermo Fisher Scientific, Inc.). After $48 \mathrm{~h}$, the luciferase activity was measured using the dual-luciferase reporter system (Promega Corporation). Firefly luciferase activity was normalized to Renilla luciferase gene activity. 

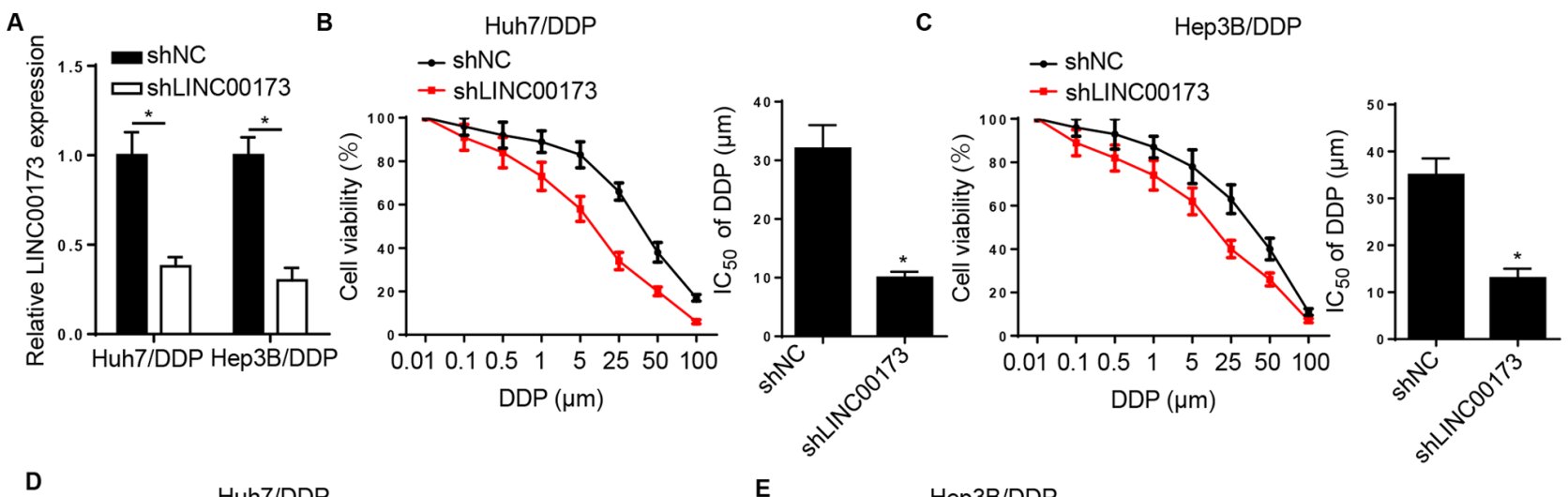

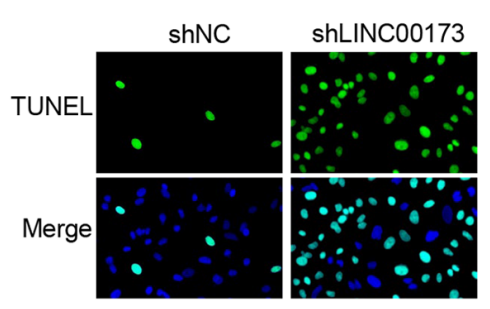

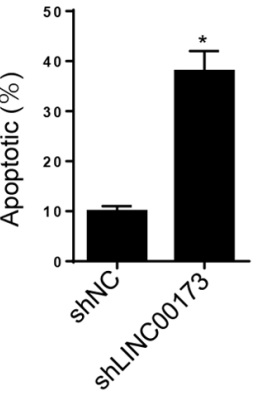

Hep3B/DDP
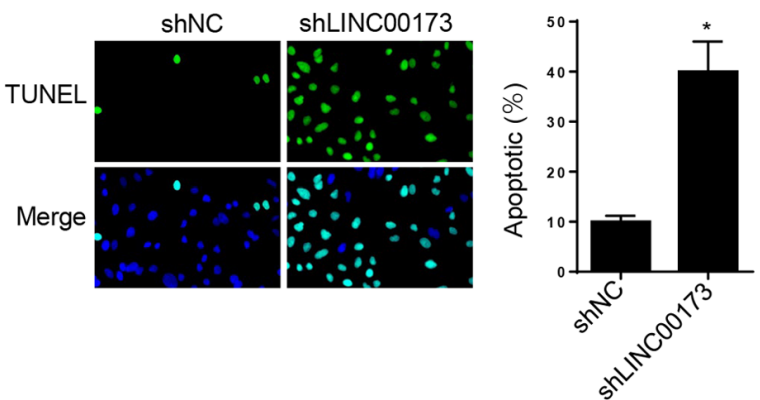

Figure 2. LINC00173-knockdown increases the sensitivity in DDP-resistant HCC cells to DDP. (A) Reverse transcription-quantitative PCR was used to determine relative LINC00173 expression levels in Huh7/DDP and Hep3B/DDP cells transfected with shNC and shLINC00173. Cell Counting Kit-8 analysis was used to determine the $\mathrm{IC}_{50}$ values of (B) Huh7/DDP and (C) Hep3B/DDP cells treated with increasing concentrations of DDP, and transfected with shNC and shLINC00173. TUNEL analysis (magnification, $x 100$ ) showed the apoptosis of (D) Huh7/DDP and (E) Hep3B/DDP cells transfected with shNC and

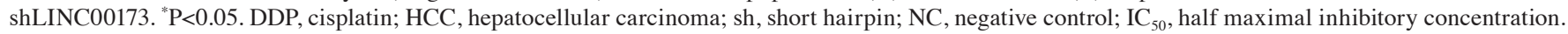

Statistical analysis. The results are presented as the mean \pm standard deviation and all experiments were repeated in triplicate. Statistical analysis was performed using SPSS software (version 19.0; IBM Corp.). Comparisons of parameters between HCC tissues and adjacent normal tissues, or experimental and control groups, were performed using the paired or unpaired Student's t-test, respectively. Comparisons among multiple groups were performed by one-way analysis of variance followed by Tukey's test. Pearson's correlation analysis was used to determine correlations between gene expression levels, and Kaplan-Meier analysis was used to estimate overall survival. $\mathrm{P}<0.05$ was considered to indicate a statistically significant difference.

\section{Results}

LINC00173 expression is increased in DDP-resistant HCC tissues and cell lines. RT-qPCR analysis demonstrated that LINC00173 expression was elevated in HCC tissues compared with adjacent normal tissues (Fig. 1A). In addition, LINC00173 expression in DDP-resistant HCC tissues was markedly higher compared with that in DDP-sensitive HCC tissues (Fig. 1B). The CCK- 8 assay indicated that the $\mathrm{IC}_{50}$ of DDP was markedly increased in Huh7/DDP and Hep3B/DDP cells, suggesting that DDP-resistant HCC cell lines were successfully established (Fig. 1C and D). Furthermore, LINC00173 expression was markedly upregulated in the DDP-resistant Huh7 and Hep3B cell lines (Huh7/DDP and Hep3B/DDP) compared with that in parental Huh7 and Hep3B cells (Fig. 1E and F). Additionally, patients with HCC and high LINC00173 expression levels exhibited shorter overall survival times (Fig. 1G). These data indicated that LINC00173 upregulation may be associated with DDP resistance in HCC.

LINC00173 knockdown increases the DDP sensitivity of DDP-resistant HCC cells. To determine the effects of LINC00173 on DDP resistance in HCC, Huh7/DDP and Hep3B/DDP cells were transfected with shNC and shLINC00173. RT-qPCR analysis indicated that LINC00173 silencing downregulated LINC00173 expression in DDP-resistant HCC cells (Fig. 2A). Furthermore, the DDP sensitivity of DDP-resistant HCC cells was enhanced by LINC00173 knockdown (Fig. 2B and C). Additionally, TUNEL analysis revealed that silencing of LINC00173 markedly increased the apoptotic rates of Huh7/DDP and Hep3B/DDP cells (Fig. 2D and E). Taken together, the aforementioned data indicated that LINC00173 knockdown increased the sensitivity of DDP-resistant HCC cells to DDP.

miR-641 is a target of LINC00173 in HCC cells. The StarBase website predicted that LINC00173 possessed a binding site for miR-641 (Fig. 3A). Then, RT-qPCR analysis revealed that miR-641 expression was increased in Huh7/DDP and Hep3B/DDP cells transfected with miR-641 mimics (Fig. 3B). A luciferase reporter assay was performed to confirm the interaction between LINC00173 and miR-641, and the results indicated that overexpression of miR-641 decreased the luciferase activity of LINC00173-wt 
A

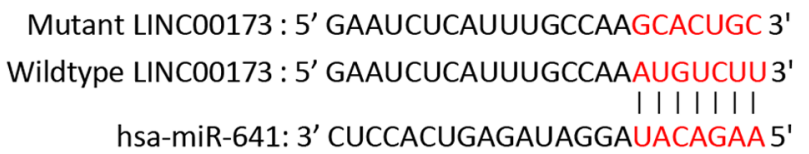

D

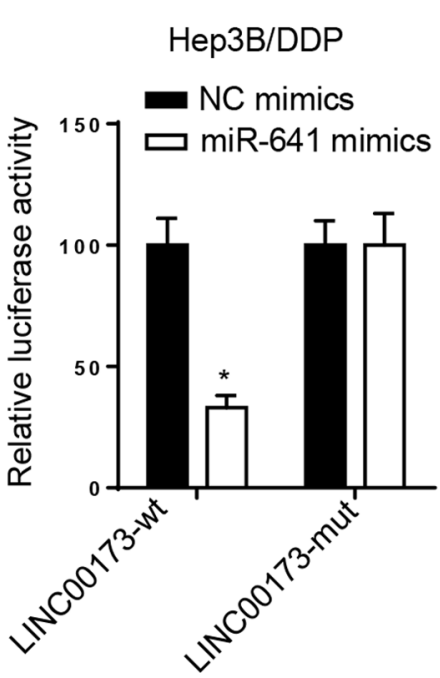

F

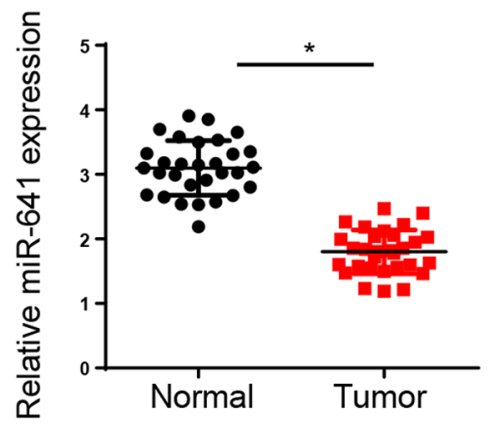

B

E

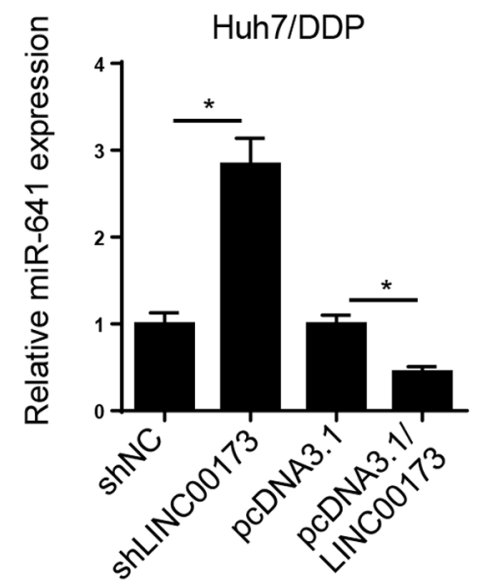

G

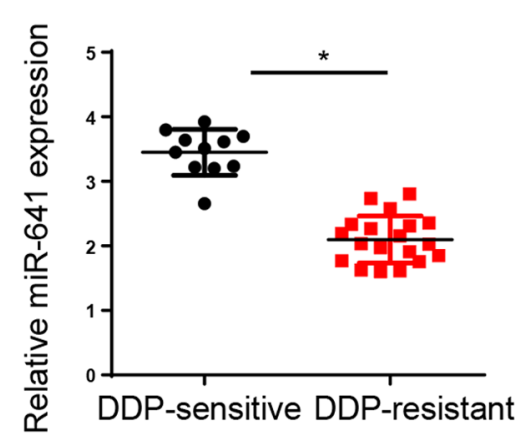

C
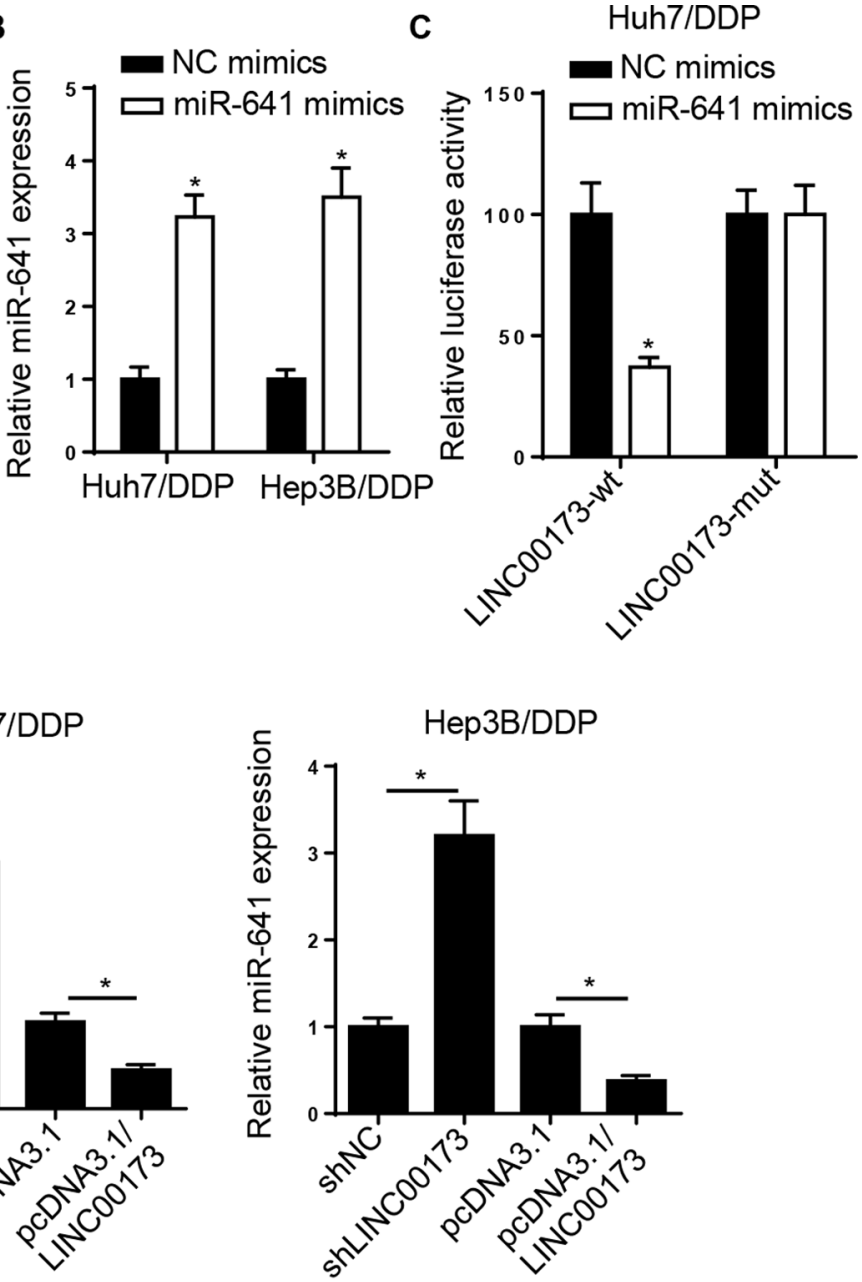

H

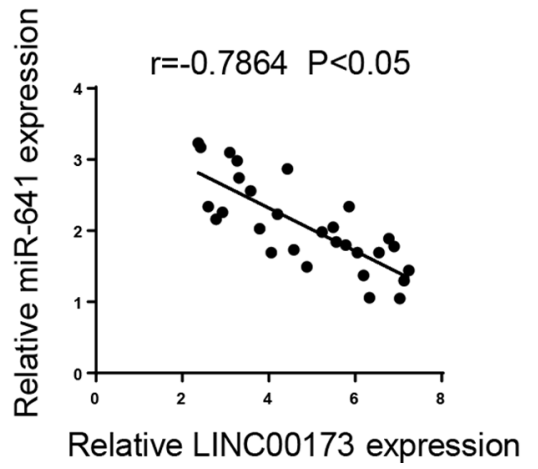

Figure 3. miR-641 is a target of LINC00173 in HCC cells. (A) StarBase was used to predict potential binding sites between LINC00173 and miR-641. (B) RT-qPCR analysis of relative miR-641 expression in Huh7/DDP and Hep3B/DDP cells transfected with NC or miR-641 mimics. Luciferase activity of LINC00173-wt or LINC00173-mut in (C) Huh7/DDP and (D) Hep3B/DDP cells transfected with NC or miR-641 mimics. (E) RT-qPCR analysis of relative miR-641 expression in Huh7/DDP and Hep3B/DDP cells transfected with shLINC00173, shNC, pcDNA3.1/LINC00173 and pcDNA3.1. Relative miR-641 expression in (F) HCC tissues and (G) DDP-resistant HCC tissues was determined by RT-qPCR. (H) Pearson's correlation analysis revealed a negative correlation between miR-641 and LINC00173 expression in HCC tissues. ${ }^{*} \mathrm{P}<0.05$. miR, microRNA; HCC, hepatocellular carcinoma; RT-qPCR, reverse transcription-quantitative PCR; DDP, cisplatin; wt, wild-type; mut, mutant; NC, negative control; sh, short hairpin.

in Huh7/DDP and Hep3B/DDP cells, but had no effect on the LINC00173-mut (Fig. 3C and D). Furthermore, the results of the RT-qPCR analysis revealed that LINC00173 depletion increased the expression of miR-641, while LINC00173 overexpression decreased miR-641 expression in DDP-resistant HCC cells (Fig. 3E). In addition, the expression of miR-641 was found to be low in HCC tissues compared with adjacent-normal tissues, particularly in DDP-resistant HCC tissues (Fig. 3F and G). Furthermore, Pearson's correlation analysis revealed that LINC00173 expression was inversely correlated with miR-641 expression in HCC tissues (Fig. $3 \mathrm{H}$ ). These results confirmed that LINC00173 inhibited the expression of miR-641 by direct interaction in DDP-resistant HCC cells. 

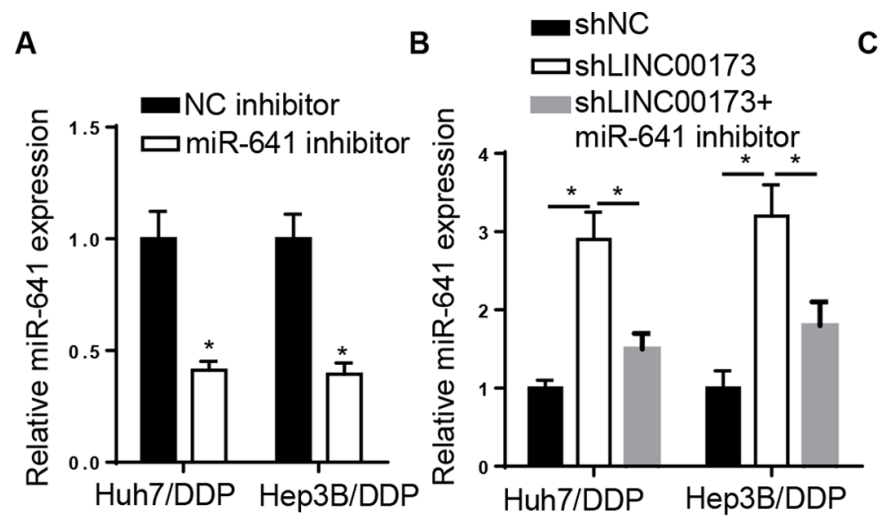

C
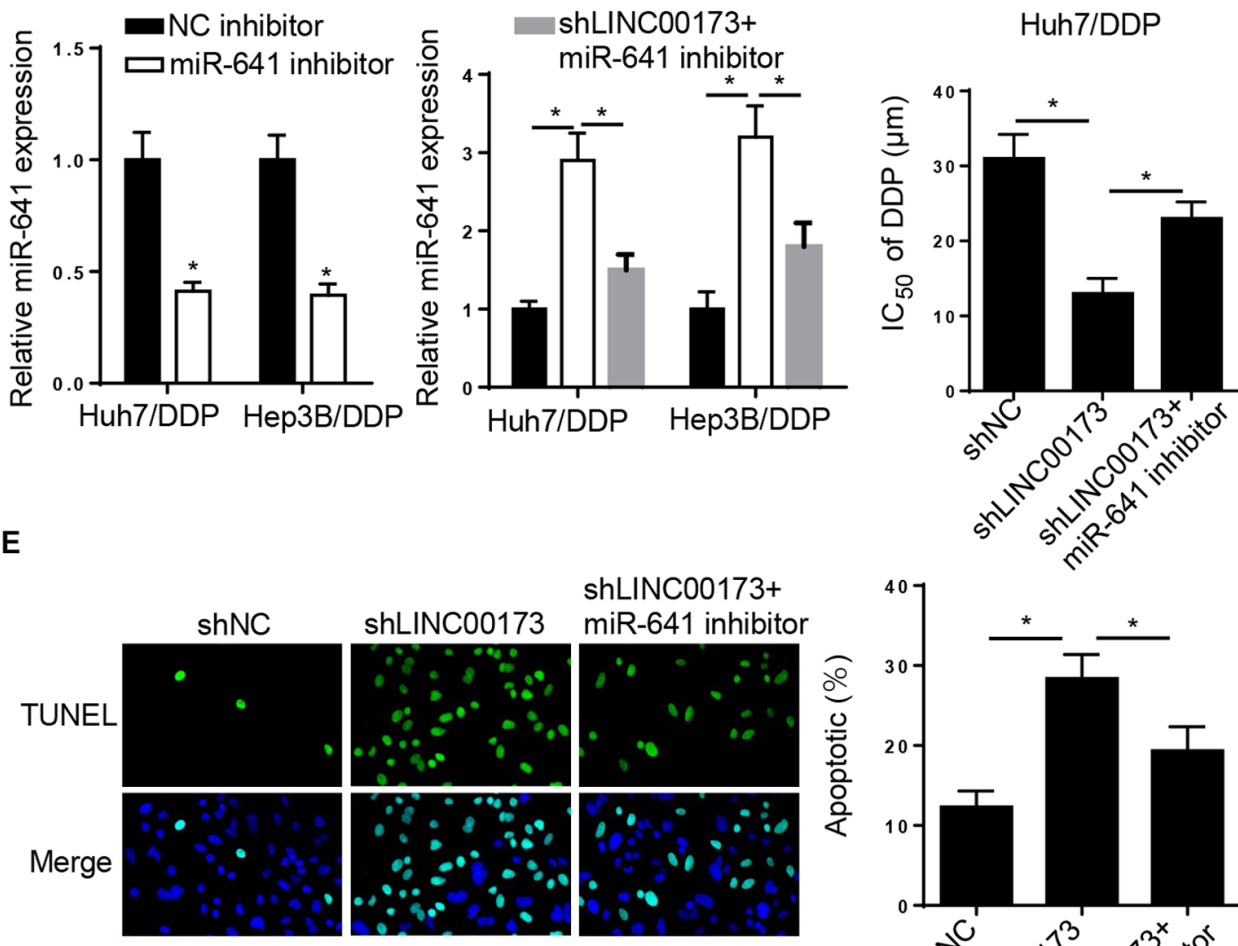

Huh7/DDP

$\mathbf{F}$

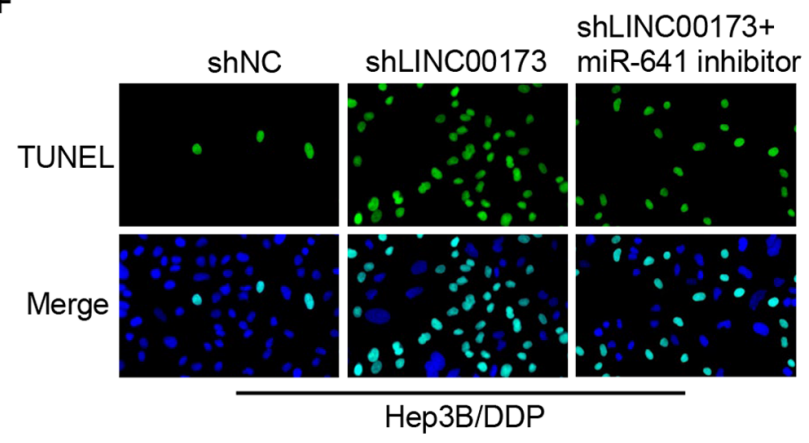

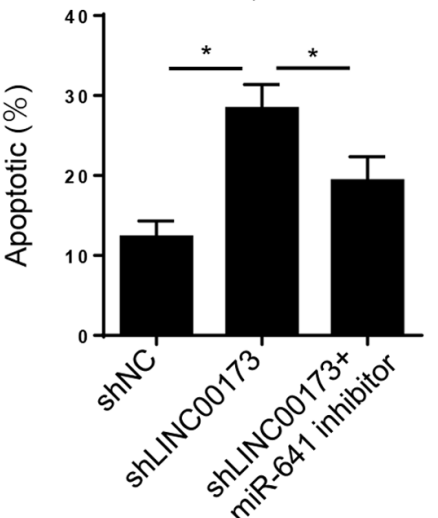

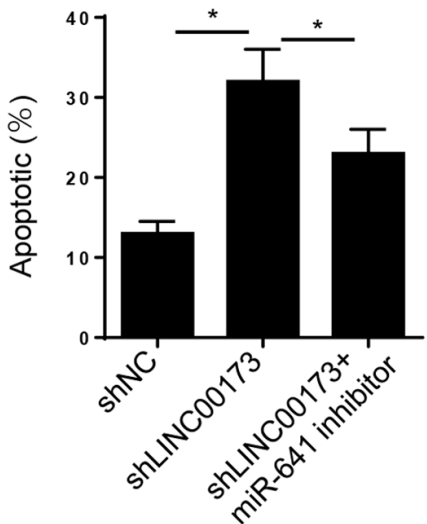

Figure 4. LINC00173-knockdown decreases DDP resistance in DDP-resistant HCC cells by targeting miR-641. (A) RT-qPCR analysis of relative miR-641 expression in Huh7/DDP and Hep3B/DDP cells transfected with the NC inhibitor or miR-641 inhibitor. (B) RT-qPCR analysis of relative miR-641 expression in Huh7/DDP and Hep3B/DDP cells transfected with shNC, shLINC00173 or shLINC00173+miR-641 inhibitor. Cell Counting Kit-8 analysis to determine the $\mathrm{IC}_{50}$ value of (C) Huh7/DDP and (D) Hep3B/DDP cells transfected with shNC, shLINC00173 or an shLINC00173+miR-641 inhibitor. TUNEL analysis (magnification, x100) revealed the apoptosis of (E) Huh7/DDP and (F) Hep3B/DDP cells transfected with shNC, shLINC00173 or shLINC00173+miR-641 inhibitor. "P<0.05. DDP, cisplatin; miR, microRNA; HCC, hepatocellular carcinoma; RT-qPCR, reverse transcription-quantitative PCR; NC, negative control; sh, short hairpin; $\mathrm{IC}_{50}$, half maximal inhibitory concentration.

LINC00173-knockdown increases DDP sensitivity in DDP-resistant HCC cells by targeting miR-641. Rescue experiments were performed to investigate the role of miR-641 in LINC00173-mediated DDP resistance in HCC. RT-qPCR analysis indicated that miR-641 expression was reduced in Huh7/DDP and Hep3B/DDP cells transfected with the miR-641 inhibitor (Fig. 4A). Furthermore, the level of miR-641 was markedly elevated in Huh7/DDP and Hep3B/DDP cells transfected with shLINC00173, which was reversed by the miR-641 inhibitor (Fig. 4B). Furthermore, the
CCK- 8 assay revealed that LINC00173 interference decreased the $\mathrm{IC}_{50}$ of DDP in Huh7/DDP and Hep3B/DDP cells, which was reversed by miR-641 inhibition (Fig. 4C and D). TUNEL analysis indicated that LINC00173-knockdown promoted the apoptosis of Huh7/DDP and Hep3B/DDP cells, while miR-641-knockdown counteracted this effect (Fig. 4E and F). In summary, these findings indicated that LINC00173-knockdown increased the sensitivity of DDP-resistant HCC cells to DDP by regulating miR-641 expression. 
A

Mutant RAB14 : 5' ACUUUUUACUGCCUCAUGACUGG 3' Wildtype RAB14: $5^{\prime}$ ACUUUUUACUGCCUCAUUGUCUU3' hsa-miR-641: 3' CUCCACUGAGAUAGgAUACAGAA5'

D

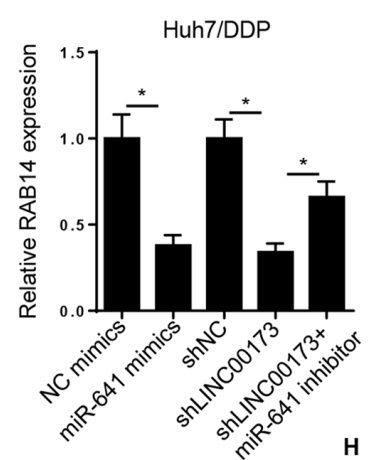

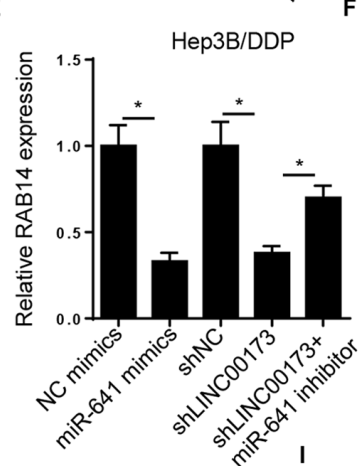

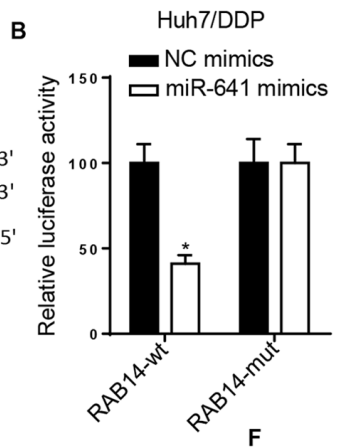

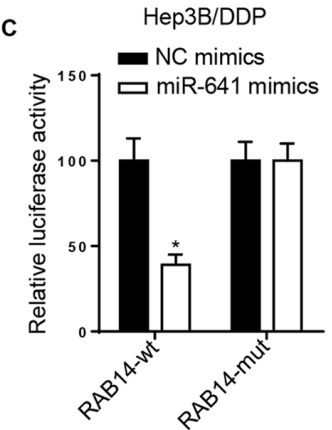
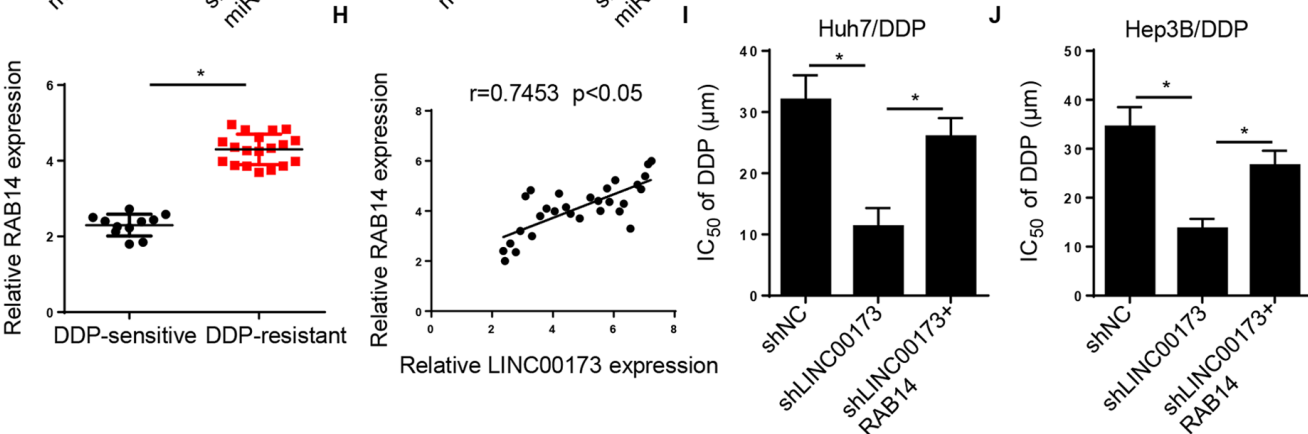

K
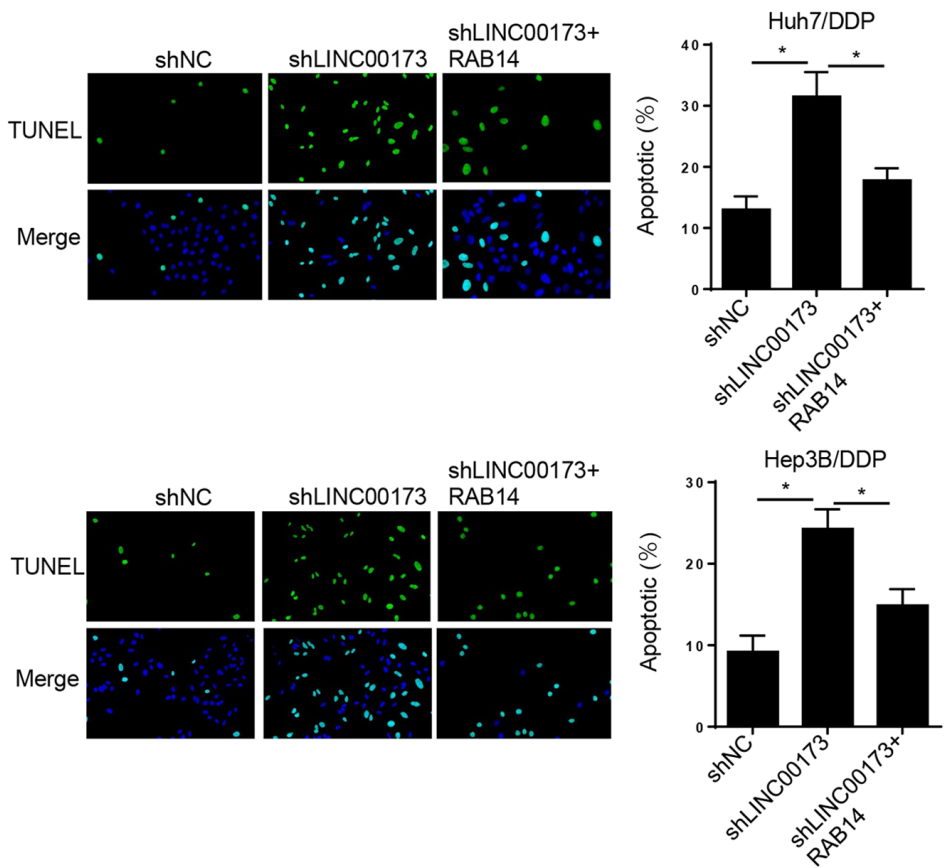

Figure 5. LINC00173 induces DDP resistance in HCC cells by modulating the miR-641/RAB14 axis. (A) StarBase was used to predict potential binding sites between RAB14 and miR-641. Luciferase activity of RAB14-wt or RAB14-mut in (B) Huh7/DDP and (C) Hep3B/DDP cells transfected with NC mimics and miR-641 mimics. RT-qPCR showed the relative RAB14 expression in (D) Huh7/DDP and (E) Hep3B/DDP cells transfected with NC mimics, miR-641 mimics, shNC, shLINC00173 or shLINC00173+miR-641 inhibitor. RT-qPCR showed the relative miR-641 expression in (F) HCC tissues and (G) DDP-resistant HCC tissues. (H) Pearson's correlation analysis revealed a positive correlation between RAB14 and LINC00173 expression in HCC tissues. Cell Counting Kit-8 analysis was used to determine the $\mathrm{IC}_{50}$ values of (I) Huh7/DDP and (J) Hep3B/DDP cells transfected with shNC, shLINC00173 or shLINC00173+pcDNA3.1/RAB14. TUNEL analysis (magnification, x100) of the apoptosis of (K) Huh7/DDP and (L) Hep3B/DDP cells transfected with shNC, shLINC00173 or shLINC00173+pcDNA3.1/RAB14. *P<0.05. DDP, cisplatin; HCC, hepatocellular carcinoma; miR, microRNA; wt, wild-type; mut, mutant; NC, negative control; sh, short hairpin (RNA); RT-qPCR, reverse transcription-quantitative PCR; IC ${ }_{50}$, half maximal inhibitory concentration. 
LINC00173 enhances DDP resistance in HCC cells by regulating the miR-641/RAB14 axis. As shown in Fig. 5A, the StarBase website predicted that RAB14 was a potential target of miR-641. A luciferase reporter assay revealed that miR-641 overexpression decreased the luciferase activity of LINC00173-wt in DDP-resistant HCC cells, while that of LINC00173-mut exhibited no significant change (Fig. 5B and C). Of note, RAB14 expression was reduced by miR-641 overexpression or LINC00173-knockdown, and the changes caused by LINC00173-knockdown were reversed by miR-641 inhibition (Fig. 5D and E). In addition, the level of RAB14 was increased in HCC tissues and in DDP-resistant HCC tissues (Fig. 5F and G). A positive correlation between LINC00173 and RAB14 expression was also observed in HCC tissues (Fig. 5H). The CCK-8 assay demonstrated that transfection of pcDNA3.1/RAB14 recovered the inhibitory effect of LINC00173-knockdown on the $\mathrm{IC}_{50}$ value of DDP in Huh7/DDP and Hep3B/DDP cells (Fig. 5I and J). Addition of RAB14 neutralized the promoting effect of LINC00173-knockdown on the apoptosis of Huh7/DDP and Hep3B/DDP cells (Fig. 5K and L). Collectively, these findings indicated that LINC00173 contributed to DDP resistance in HCC cells through sponging miR-641 and upregulating RAB14.

\section{Discussion}

Chemoresistance severely compromises therapeutic efficacy in patients with HCC. Thus, it is crucial to identify the molecular mechanisms underlying HCC chemoresistance and to investigate new therapeutic strategies for HCC. In the present study, it was observed that silencing of LINC00173 improved the sensitivity of DDP-resistant HCC cells by modulating the miR-641/RAB14 axis, suggesting that LINC00173 may be an effective treatment target to prevent or reverse drug resistance.

Numerous studies have reported that lncRNAs are key regulators of chemoresistance in human cancers $(20,21)$. Dysregulated LINC00173 expression was found to be implicated in drug resistance in cancer. For example, lncRNA LINC00173 promoted colorectal cancer cell metastasis and chemoresistance via modulation of the miR-765/PLP2 pathway (22). LINC00173 facilitated the chemoresistance and progression of small-cell lung cancer by targeting miR-218 and regulating Etk expression (23). The present study focused on the role and mechanism of action of LINC00173 in DDP-resistant HCC. The results indicated that LINC00173 expression was elevated in HCC tissues and DDP-resistant HCC cells. Furthermore, LINC00173 knockdown enhanced the DDP sensitivity of DDP-resistant HCC cells. These findings indicated that LINC00173 induced DDP resistance in HCC cells.

A large number of studies have revealed that lncRNAs function as competing endogenous RNAs, exerting their regulatory effects on tumorigenesis by sponging miRNAs, which reverses the inhibitory effects on their targets $(24,25)$. miR-641 has been reported to act as a tumor suppressor in various cancer types. For example, miR-641 suppressed lung cancer cell proliferation and induced apoptosis by regulating Programmed cell death protein 4 (26). miR-641 also inhibited cervical cancer progression and promoted apoptosis by targeting zinc finger E-box-binding homeobox 1 (27). However, the biological function of miR-641 in DDP-resistant HCC cells remained unclear. In the present study, the level of miR-641 was reduced in DDP-resistant HCC tissues and miR-641 was found to directly interact with LINC00173. Furthermore, miR-641 inhibition abolished the promoting effect of LINC00173 knockdown on the sensitivity of DDP-resistant HCC cells. These results indicated that LINC00173 enhanced DDP resistance in HCC by regulating miR-641.

RAB14, a member of the RAS oncogene family, has been reported to be implicated in the development of several types of cancer, such as cervical and gastric cancer $(28,29)$. Convincing evidence has suggested that RAB14 may promote chemotherapy resistance in several tumors $(30,31)$. For example, miR-148a enhanced sensitivity to DDP by targeting RAB14 in renal cancer cells (32). In the present study, RAB14 expression was markedly enhanced in DDP-resistant HCC tissues, and was confirmed to be a target of miR-641. Furthermore, rescue assays revealed that RAB14 overexpression partially abolished the enhanced DDP sensitivity of Huh7/DDP and Hep3B/DDP cells caused by LINC00173-knockdown. Collectively, these data confirmed that LINC00173 contributed to DDP resistance in HCC by modulating the miR-641/RAB14 axis.

However, the limitations of the present study must be addressed in future research. For example, the downstream effectors or signaling pathways associated with the LINC00173/miR-641/RAB14 axis should be further investigated, and in vivo experiments will help to fully elucidate the associated underlying mechanisms.

In conclusion, the findings of the present study demonstrated that LINC00173 promoted the DDP resistance of HCC cells via sponging miR-641 to upregulate RAB14, providing a novel therapeutic target for overcoming DDP resistance in HCC.

\section{Acknowledgements}

Not applicable.

\section{Funding}

No funding was received.

\section{Availability of data and materials}

The datasets used and/or analyzed during the present study are available from the corresponding author on reasonable request.

\section{Authors' contributions}

GZ and YD designed the study. AZ and SS performed the experiments. GZ and AZ analysed the data and prepared the figures. GZ and YD drafted, reviewed and revised the manuscript. GZ and YD confirm the authenticity of all the raw data. All authors read and approved the final manuscript.

\section{Ethics approval and consent to participate}

The present study was approved by the Ethics Committee of Weifang People's Hospital (Weifang, China), and written 
informed consent was obtained from all subjects prior to commencement.

\section{Patient consent for publication}

Not applicable.

\section{Competing interests}

The authors declare that they have no competing interests.

\section{References}

1. Singal AG and El-Serag HB: Hepatocellular carcinoma from epidemiology to prevention: Translating knowledge into practice. Clin Gastroenterol Hepatol 13: 2140-2151, 2015.

2. Fan ST, Mau Lo C, Poon RT, Yeung C, Leung Liu C, Yuen WK, Ming Lam C, Ng KK and Ching Chan S: Continuous improvement of survival outcomes of resection of hepatocellular carcinoma: A 20-year experience. Ann Surg 253: 745-758, 2011.

3. Schmitt AM and Chang HY: Long noncoding RNAs in cancer pathways. Cancer Cell 29: 452-463, 2016.

4. Gibb EA, Brown CJ and Lam WL: The functional role of long non-coding RNA in human carcinomas. Mol Cancer 10: 38, 2011

5. Shi Y, Yang X, Xue X, Sun D, Cai P, Song Q, Zhang B and Qin L: HANR enhances autophagy-associated sorafenib resistance through miR-29b/ATG9A axis in hepatocellular carcinoma Onco Targets Ther 13: 2127-2137, 2020.

6. Guo Y, Bai M, Lin L, Huang J, An Y, Liang L, Liu Y and Huang W: LncRNA DLEU2 aggravates the progression of hepatocellular carcinoma through binding to EZH2. Biomed Pharmacother 118 : $109272,2019$.

7. Liu Z, Chen JY, Zhong Y, Xie L and Li JS: IncRNA MEG3 inhibits the growth of hepatocellular carcinoma cells by sponging miR-9-5p to upregulate SOX11. Braz J Med Biol Res 52: e8631, 2019.

8. Yang X, Sun L, Wang L, Yao B, Mo H and Yang W: LncRNA SNHG7 accelerates the proliferation, migration and invasion of hepatocellular carcinoma cells via regulating miR-122-5p and RPL4. Biomed Pharmacother 118: 109386, 2019.

9. Gu M, Zheng W, Zhang M, Dong X, Zhao Y, Wang S, Jiang H and Zheng X: LncRNA NONHSAT141924 promotes paclitaxel chemotherapy resistance through $\mathrm{p}-\mathrm{CREB} / \mathrm{Bcl}-2$ apoptosis signaling pathway in breast cancer. J Cancer 11: 3645-3654, 2020.

10. Zhang J, Zhao B, Chen X, Wang Z, Xu H and Huang B: Silence of long noncoding RNA NEAT1 inhibits malignant biological behaviors and chemotherapy resistance in gastric cancer. Pathol Oncol Res 24: 109-113, 2018

11. Zhang J, Zhou M, Zhao X, Wang G and Li J: Long noncoding RNA LINC00173 is downregulated in cervical cancer and inhibits cell proliferation and invasion by modulating the miR-182-5p/FBXW7 axis. Pathol Res Pract 216: 152994, 2020

12. Fan H, Yuan J, Li X, Ma Y, Wang X, Xu B and Li X: LncRNA LINC00173 enhances triple-negative breast cancer progression by suppressing miR-490-3p expression. Biomed Pharmacother 125: 109987, 2020.

13. Yang F, Lei $\mathrm{P}$, Zeng $\mathrm{W}, \mathrm{Gao} \mathrm{J}$ and $\mathrm{Wu} \mathrm{N}$ : Long noncoding RNA LINC00173 promotes the malignancy of melanoma by promoting the expression of IRS4 through competitive binding to microRNA-493. Cancer Manag Res 12: 3131-3144, 2020.

14. Xiao F, Li Y, Wan Y and Xue M: MircroRNA-139 sensitizes ovarian cancer cell to cisplatin-based chemotherapy through regulation of ATP7A/B. Cancer Chemother Pharmacol 81: 935-947, 2018
15. Niu YC, Tong J, Shi XF and Zhang T: MicroRNA-654-3p enhances cisplatin sensitivity by targeting QPRT and inhibiting the PI3K/AKT signaling pathway in ovarian cancer cells. Exp Ther Med 20: 1467-1479, 2020.

16. Yang S, Wang P, Wang S, Cong A, Zhang Q, Shen W, Li X, Zhang W and Han G: miRNA-181a-5p enhances the sensitivity of cells to cisplatin in esophageal adenocarcinoma by targeting CBLB. Cancer Manag Res 12: 4981-4990, 2020.

17. Zhao Y, Zheng R, Chen J and Ning D: CircRNA CDR1as/miR-641/HOXA9 pathway regulated stemness contributes to cisplatin resistance in non-small cell lung cancer (NSCLC). Cancer Cell Int 20: 289, 2020.

18. van Persijn van Meerten EL, Gelderblom $\mathrm{H}$ and Bloem JL: RECIST revised: Implications for the radiologist. A review article on the modified RECIST guideline. Eur Radiol 20: 1456-1467, 2010.

19. Livak KJ and Schmittgen TD: Analysis of relative gene expression data using real-time quantitative PCR and the 2(-Delta Delta C(T)) method. Methods 25: 402-408, 2001

20. Wang SY, Wang X and Zhang CY: LncRNA SNHG7 enhances chemoresistance in neuroblastoma through cisplatin-induced autophagy by regulating miR-329-3p/MYO10 axis. Eur Rev Med Pharmacol Sci 24: 3805-3817, 2020.

21. Xu Q, Lin YB, Li L and Liu J: LncRNA TLR8-AS1 promotes metastasis and chemoresistance of ovarian cancer through enhancing TLR8 mRNA stability. Biochem Biophys Res Commun 526: 857-864, 2020.

22. Yu Y, Lu X, Yang C and Yin F: Long noncoding RNA LINC00173 contributes to the growth, invasiveness and chemo-resistance of colorectal cancer through regulating miR-765/PLP2 axis. Cancer Manag Res 12: 3363-3369, 2020

23. Zeng F, Wang Q, Wang S, Liang S, Huang W, Guo Y, Peng J, Li M, Zhu W and Guo L: Linc00173 promotes chemoresistance and progression of small cell lung cancer by sponging miR-218 to regulate Etk expression. Oncogene 39: 293-307, 2020.

24. Wang Y, Hou J, He D, Sun M, Zhang P, Yu Y and Chen Y: The emerging function and mechanism of ceRNAs in cancer. Trends Genet 32: 211-224, 2016.

25. Mercer TR, Dinger ME and Mattick JS: Long non-coding RNAs: Insights into functions. Nat Rev Genet 10: 155-159, 2009.

26. Zhou J, Li H, Li N, Li X, Zhang H, Song Q and Peng M: MicroRNA-641 inhibits lung cancer cells proliferation, metastasis but promotes apoptosis in cells by targeting PDCD4. Int J Clin Exp Pathol 10: 8211-8221, 2017.

27. Yao R, Zheng H, Wu L and Cai P: miRNA-641 inhibits the proliferation, migration, and invasion and induces apoptosis of cervical cancer cells by directly targeting ZEB1. Onco Targets Ther 11: 8965-8976, 2018.

28. Yang J, Liang B and Hou S: TMPO-AS1 promotes cervical cancer progression by upregulating RAB14 via sponging miR-577. J Gene Med 21: e3125, 2019.

29. Guo B, Wang W, Zhao Z, Li Q, Zhou K, Zhao L, Wang L, Yang J and Huang C: Rab14 Act as oncogene and induce proliferation of gastric cancer cells via AKT signaling pathway. PLoS One 12: e0170620, 2017.

30. Zhai Y, Liu M and Zheng Y: MicroRNA-451 dictates the anoikis resistance of osteosarcoma by targeting Rab14. Int J Clin Exp Pathol 10: 10989-10997, 2017.

31. Ge J and Ge C: Rab14 overexpression regulates gemcitabine sensitivity through regulation of $\mathrm{Bcl}-2$ and mitochondrial function in pancreatic cancer. Virchows Arch 474: 59-69, 2019.

32. Kim EA, Kim TG, Sung EG, Song IH, Kim JY, Doh KO and Lee TJ: miR-148a increases the sensitivity to cisplatin by targeting Rab14 in renal cancer cells. Int J Oncol 50: 984-992, 2017.

This work is licensed under a Creative Commons Attribution-NonCommercial-NoDerivatives 4.0 International (CC BY-NC-ND 4.0) License. 